Figure 1 Embryonic macrophages selectively maintain mitochondrial membrane polarization following induction of apoptosis. (a) S2 cells stained with CMXRos: untreated, mock treated, FCCP treated, UV-irradiated. (b, c) Stage 13 embryos stained with CMXRos and imaged live through the vitelline membrane. (b) CyO/CyO, (c) hs-hid/CyO. Embryos in ( $\mathbf{b}$ and $\mathbf{c}$ ) were subjected to 60 min of heat shock before staining. Embryos in ( $\mathbf{b}$ and $\mathbf{c}$ ) are siblings and were prepared and imaged together. Genotypes were determined by the intensity of green fluorescent protein (GFP) from a kruppel-GFP CyO balancer. (d) Head region of embryo shown in (c) showing CMXRos-positive macrophages in a field of CMXRos-negative cells. (e) hs-hid/CyO embryo subjected to 60 min of heat shock and labeled with CMXRos and anti-Crq to confirm the identity of CMXRos-positive cells. Image is of the extreme anterior portion of the embryonic head. (f) TEM of $90 \mathrm{~nm}$ thin section through the head region of a stage $13 \mathrm{hs}$-hid embryo subjected to $60 \mathrm{~min}$ of heat shock before fixation. Low-magnification image of region showing a healthy macrophage (large cell, boxed) surrounded by apoptotic corpses (small cell boxed; asterisks). Higher magnification images of boxed cells are shown in Supplementary Figure 1

irradiation and a recovery period of $6 \mathrm{~h}$ (Figure 1a). However, this is not the case with Drosophila cells in general. Embryos subjected to ectopic hid expression (Figure 1c-e) or UV irradiation (not shown) rapidly lose the capacity to take up CMXRos in nearly all cells. Within $1 \mathrm{~h}$ of the initiation of either signal, nearly all cells of the embryo fail to stain with CMXRos. The notable exception is macrophages, which continue to stain at high levels whereas other cell types do not (Figure 1c-d). These cells are identifiable as macrophages by their stereotypic distribution and enormous size, and by doubly labeling for Croquemort (Figure 1e), a macrophage-specific cell surface marker. ${ }^{11,12}$ Identical results were obtained using the mitochondrial marker TMRE (not shown).

These findings suggest that macrophages have the capacity to remain healthy in the presence of apoptotic signals that readily affect other cell types. We addressed this possibility by examining the ultrastructure of macrophages in apoptotic embryos by transmission electron microscopy. We found that after $1 \mathrm{~h}$ of heat shock-induced hid expression, macrophages in the embryonic head showed no overt signs of apoptosis, while surrounded by apoptotic corpses derived from other cell types (Figure 1f; Supplementary Figure 1). Macrophage chromatin remained uncondensed with the nuclear envelope intact, and membranebound organelles including mitochondria, Golgi and endoplasmic reticulum were clearly visible in the cytoplasm. By contrast, surrounding corpses showed condensed chromatin, degeneration of the nuclear envelope and loss of subcellular organelles (Figure 1f; Supplementary Figure 1). These results are consistent with observations by us and others that following exposure to UV irradiation, Croquemort-positive cells in the embryo become quite large owing to multiple engulfment events ( $R$ Hays, unpublished finding). ${ }^{11}$ One would expect macrophages to undergo apoptosis like any other cell type, having been exposed to the same dose of radiation. However, they survive to engulf corpses for a period of at least several hours.

These findings suggest that macrophages are uniquely resistant to apoptosis among embryonic cell types, and that S2 cells, an embryonic macrophage line, is not a suitable general model for Drosophila cell death. They further suggest that apoptosis regulation in Drosophila has more in common with vertebrate systems than previously appreciated, and re-shape a large segment of current Drosophila apoptosis models.

Acknowledgements. We are grateful to $\mathrm{A}$ Ezekowitz and $\mathrm{R}$ Ward for reagents. This work was supported by NIH 2 P20 RR016475-04.

\section{JC Means ${ }^{1}$ and $R$ Hays $^{1}$}

Division of Biological Sciences, University of Kansas, 1200 Sunnyside Avenue, Lawrence, KS, USA

Corresponding author: R Hays, Division of Biological Sciences, University of Kansas, 1200 Sunnyside Avenue, Lawrence, KS 66045, USA.

Tel. + 1-785-864-5744; Fax; + 1-785-864-5294;

E-mail: rebahays@ku.edu

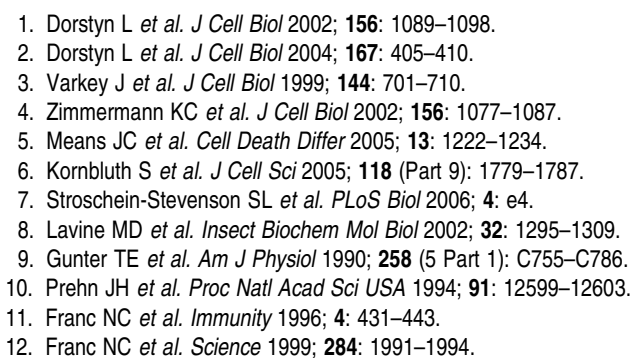

Supplementary Information accompanies the paper on Cell Death and Differentiation website (http://www.nature.com/cdd)

\title{
Cavitation of embryoid bodies requires optimal oxidative phosphorylation and AIF
}

\author{
Cell Death and Differentiation (2007) 14, 385-387. doi:10.1038/sj.cdd.4402041; published online 29 September 2006
}

\section{Dear Editor,}

Apoptosis inducing factor (AIF), which is encoded by the $p c d 8$ gene on the $\mathrm{X}$ chromosome, is a protein that is normally present in mitochondria. ${ }^{1,2}$ The AIF flavoprotein can participate in the scavenging of reactive oxygen species, ${ }^{3}$ and 
a
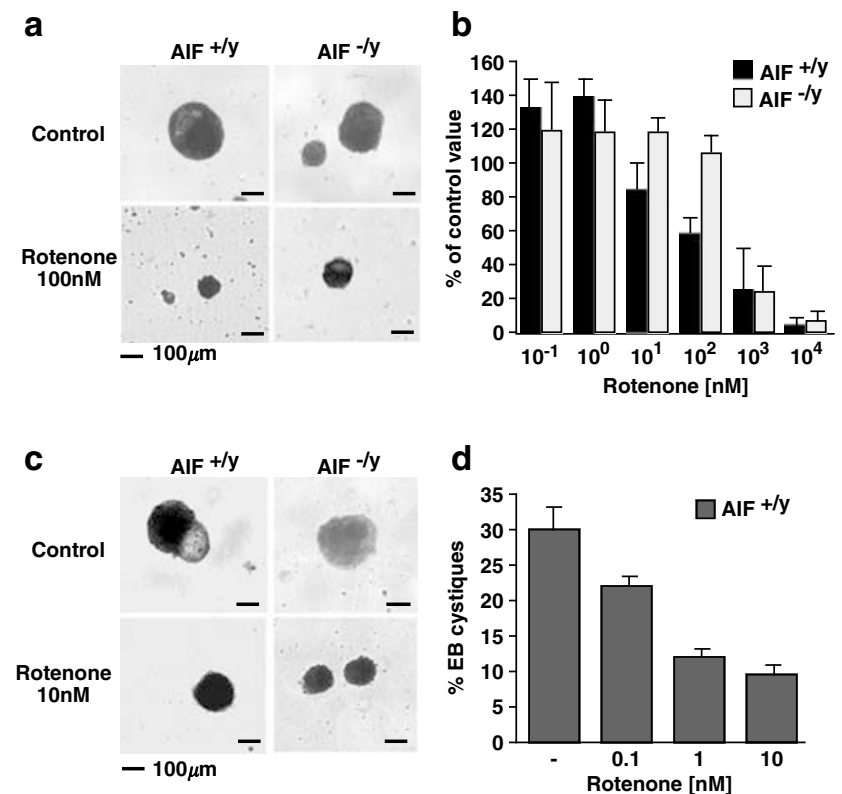

Figure 1 Response of ES cells to complex inhibition by rotenone. (a, b). Reduced vulnerability of AIF-/y cells to complex I inhibition. Embryonic bodies (EBs) derived from AIF-/y or AIF + /y ES cells were cultured for three days in the presence of the indicated concentration of rotenone $(100 \mathrm{nM}$ in (a). Representative microphotographs are shown in (a). Note that rotenone-treated $\mathrm{AIF}+/ \mathrm{y}$ EBs form much more debris than control EBs). The frequency of viable cells was determined after dissociation of the EBs using a cytofluorometric method in B. What does B actually show. To generate embryoid bodies (EBs), ES cells were cultured in Iscove's modified Dulbecco's medium (IMDM, Invitrogen) supplemented with 15\% FCS (Hyclone, Perbio), 1\% L-Glutamin (Invitrogen), 1\% Penicillin/Streptomycin (Invitrogen), $50 \mu \mathrm{g} / \mathrm{ml}$ ascorbic acid (Sigma), $450 \mu \mathrm{M}$ monothioglycerol (Sigma) and $200 \mu \mathrm{g} / \mathrm{ml}$ iron-saturated transferrin (Sigma). Undifferentiated ES cells $\left(4.5 \times 10^{4} \mathrm{cell} / \mathrm{ml}\right)$ were seeded in bacterial grade dishes (Nunc) and incubated at $37^{\circ} \mathrm{C}$ under a low $\mathrm{O}_{2}$ concentration $\left(7 \% \mathrm{O}_{2}\right)$. By 3 days of differentiation, EBs were treated with various concentration of rotenone (Sigma) or with vehicle alone. Embryoid bodies were then allowed to grow for further 3 days before analysis by flow cytometry. EBs were collected, dissociated in non-enzymatic Cell Dissociation Solution (Sigma), and subjected to the quantitation of viable PI-negative, $\mathrm{DIOC}_{6}(3)$-positive cells on a FACS Sort cytofluorometer (Becton and Dickinson). (c, d). Inhibition of cavitation by rotenone. ES cells with the indicated genotypes were cultured as in (a), with the difference that cultures were allowed to proceed until day 10 . Representative pictures showing one cystic EB (formed from AIF + /y ES cells) and compact EBs obtained after inhibition with rotenone (10 nM) in AlF + /y ES cells or AIF-ly ES cells are shown in $\mathbf{c}$ and the percentage of cystic embryonic bodies obtained in the presence of different concentrations of rotenone is quantified in (d). As reported previously, the $\mathrm{AIF}^{-Y}$ ES cells did not cavitate. All the experiments have been reproduced at least three times, with similar results

normal AIF levels are required for the assembly and/or maintenance of the complex I of the respiratory chain. ${ }^{4}$ Thus, AIF-deficient human and mouse cells manifest a reduced abundance and function of complex I that may results from a deficient antioxidant defense in mitochondria. ${ }^{5}$ Upon outer mitochondrial membrane permeabilization, a process that generally occurs during apoptosis, ${ }^{6}$ AIF translocates from mitochondria via the cytosol to the nucleus where it participates in apoptotic chromatin condensation, presumably through a direct interaction with DNA. ${ }^{4}$ AIF can also participate in apoptotic chromatinolysis, presumably by recruiting catabolic enzymes to the so-called degradosome. ${ }^{7}$

The AIF gene has been knocked out or knocked down in several species including in human cell lines, ${ }^{8}$ mice, ${ }^{9}$
Caenorhabditis elegans ${ }^{10}$ and Saccharomyces cerevisiae. ${ }^{11}$ In yeast, the knockout of AIF leads to a partial resistance to oxidative stress and influences replicative aging. ${ }^{11}$ In nematodes, the knockdown of AIF leads to reduction of developmental cell death, in particular, in animals that bear loss-of-function mutations of caspases. ${ }^{10}$ In mammalian cells, removal of AIF can reduce apoptosis in some particular settings, although there is no general apoptosis defect. $^{2,8,9}$ Thus, knockdown of AIF protects differentiated PC12 cells against the neurotoxin 1-methyl-4-phenylpyridinium, ${ }^{12}$ Jurkat $T$ lymphoma cells against a combination of $\gamma$-irradiation and phytosphingosin, ${ }^{13}$ erythroleukemic HEL cells against CD44 ligation, ${ }^{14}$ melanoma cells against the Raf inhibitor BAY 43-9006 (Sorafenib), ${ }^{15}$ U937 promonocytic cells against cadmium, ${ }^{16}$ and Raji $B$ lymphoma cells against UV irradiation. ${ }^{17}$ Microinjection of AIF-neutralizing antibodies can also reduce the neurotoxic effects of NMDA in primary murine cortical cultures, ${ }^{18}$ the lethal effects of PARP-1 activators in several cellular systems, ${ }^{19}$ as well as the proapoptotic effects of staurosporin on non-small cell lung carcinoma cells. ${ }^{20}$

While embryonic stem (ES) cells lacking AIF $\left(\mathrm{AIF}^{-/ y}\right.$ ) are relatively resistant against serum withdrawal-induced apoptosis, as compared to wild-type ES cells, ${ }^{9}$ they respond to most apoptosis inducers including DNA-damaging agents and tyrosine kinase inhibitors normally. According to one report, $\mathrm{AIF}^{-/ y} \mathrm{ES}$ cells fail to undergo cavitation upon culture in the absence of leukemia-inhibitory factor (LIF), ${ }^{9}$ a process that usually induces aggregation of ES cells followed by apoptosis of the cells in the inner mass. ${ }^{21}$ Cavitation is regarded as the earliest wave of programmed cell death during embryogenesis, Two recent reports based on the use of another technique of AIF inactivation (the cross of mice expressing a $\beta$-actin cre transgene with mice having a floxed AIF locus) came to the conclusion that AIF was not required for cavitation in vivo ${ }^{22}$ or in vitro ${ }^{22}$ and that the observed in vitro phenotype, the absence or presence of cavitation, was influenced by the culture conditions and/or the genetic background. ${ }^{23}$

Driven by these considerations, we addressed the question whether the defect in cavitation observed in $\mathrm{AIF}^{-/ y} \mathrm{ES}$ cells might be secondary to a defect in complex I. Hence, we cultured $\mathrm{AIF}^{+/ y}$ and $\mathrm{AIF}^{-/ y} \mathrm{ES}$ cells in the presence of variable doses of the highly specific complex I inhibitor rotenone ${ }^{24}$ for 3 days (when ES cells aggregate upon removal of LIF) or 10 days (when cavitation has occurred in normal circumstances, in wild-type embryoid bodies). Rotenone caused a dosedependent inhibition in the cellularity of 6-day-old embryoid bodies (Figure 1a, b). Of note, at a dose of 1 or $10 \mathrm{nM}$, rotenone was more toxic for $\mathrm{AlF}^{+/ y}$ than for $\mathrm{AlF}^{-/ y} \mathrm{ES}$ (Figure $1 b$ ), in line with previous observations suggesting that AIF-deficient cells compensate their partial deficiency in respiration. ${ }^{4}$ Rotenone used at doses of 1 or $10 \mathrm{nM}$ also strongly inhibited cavitation in 10-day-old AIF $^{+/ y}$ embryoid bodies (Figure 1c, d). Rotenone-treated $\mathrm{AlF}^{+/ y}$ and $\mathrm{AlF}^{-/ y}$ embryoid bodies exhibited a similar phenotype, without cavitation. Thus, specific inhibition of the complex I of the respiratory chain is sufficient to perturb cellular metabolism and/or the signal transduction pathways leading to cavitationassociated apoptosis. As a word of caution, it should be 
mentioned that embryonic bodies cultured in the presence of rotenone were smaller than those lacking AIF, suggesting subtle differences between the acute complex I defect induced by rotenone (that might affect homeostatic systems in the cell other than respiration) and the chronic defect induced by the AIF knockout.

These results illustrate the difficulty to separate the two functions of AIF, first as a mitochondrial redox enzyme required for normal respiratory function and, second, as a factor that can participate in the apoptotic execution phase, after its translocation to the nucleus. The observation that AIF participates in cavitation-associated apoptosis ${ }^{9}$ implicitly suggested that cavitation would depend on the lethal action of AIF. Based on recent insights on AIF biology, however, it is possible that at least some of the lethal processes that were interrupted by the removal of AIF from the experimental system actually were suppressed due to defective bioenergetic and/or redox metabolism. As a result, we recommend the use of rotenone as an internal control in experiments, in which the apoptosis-modulatory effects of human or mouse AIF are assessed. Only when rotenone fails to modify the apoptotic response, the interpretation that the lethal (rather than the vital) action of AIF is involved in the process can be maintained.

Knock-in mutations that affect only the lethal function of AIF yet do not interfere with its metabolic activity in mitochondria are being prepared in several laboratories, ${ }^{2}$ and the use of such mutants will yield important insights into the contribution of AIF to lethal signal transduction processes. Such knock-in mutations will also help to decipher the contribution of AIF, alone or in combination with mutations of the apoptosome components, to programmed cell death in physiological and pathological cell death.
Acknowledgements. This work has been supported by a special grant from the Ligue Nationale contre le Cancer (to GK and ND), as well as grants from the European Commission (Trans-Death to GK). OF received a fellowship from the Ligue contre le Cancer.

\section{O Feraud ${ }^{1}$, N Debili $^{1}$, JM Penninger ${ }^{2}$ and G Kroemer $^{\star, 3}$}

1 INSERM U790, Institut Gustave Roussy PR1, Villejuif, France;

2 IMBA, Institute of Molecular Biotechnology of the Austrian Academy of Sciences, Vienna, Austria and

${ }^{3}$ CNRS-FRE2939, Institut Gustave Roussy, Villejuif, France

* Corresponding author: G Kroemer, CNRS-FRE2939 Institut Gustave Roussy, Pavillon de Recherche 139 rue Camille-Desmoulins F-94805 Villejuif, France. Tel: + 331421160 46; Fax: + 331421160 47;

E-mail: kroemer@igr.fr

1. Lorenzo HK et al. Cell Death Differ 1999; 6: 516-524.

2. Modjtahedi N et al. Trends Cell Biol 2006; 16: 264-272.

3. Klein JA, Ackerman SL. J Clin Invest 2003; 111: 785-793.

4. Vahsen $\mathrm{N}$ et al. EMBO J 2004; 23: 4679-4689.

5. Apostolova $\mathrm{N}$ et al. Cell Death Differ 2006; 13: 354-357.

6. Green DR, Kroemer G. Science 2004; 305: 626-629.

7. Parrish JZ, Xue D. Mol Cell 2003; 11: 987-996.

8. Urbano A et al. EMBO J 2005; 24: 2815-2826.

. Joza N et al. Nature 2001; 410: 549-554.

10. Wang $X$ et al. Science 2002; 298: 1587-1592.

11. Wissing $S$ et al. J Cell Biol 2004; 166: 969-974.

12. Liou AK et al. Faseb J 2005; 19: 1350-1352.

13. Park MT et al. Blood 2005; 105: 1724-1733.

14. Artus $\mathrm{C}$ et al. Oncogene 2006, in press.

15. Panka DJ et al. Cancer Res 2006; 66: 1611-1619.

16. Sancho $P$ et al. Apoptosis 2006; 11: 673-686.

17. Yuan CQ, Li YN, Zhang XF. Biochem Biophys Res Commun 2004; 317: 1108-1113.

18. Wang $\mathrm{H}$ et al. J Neurosci 2004; 24: 10963-10973.

19. Yu SW et al. Science 2002; 297: 259-263.

20. Gallego M-A et al. Oncogene 2004; 23: 6282-6291.

21. Coucouvanis E, Martin GR. Cell 1985; 83: 279-287.

22. Joza N et al. Mol Cell Biol 2005; 25: 10261-10272.

23. Brown D et al. Proc Natl Acad Sci USA 2006; 103: 9918-9923.

24. Chance B et al. J Biol Chem 1963; 238: 418-431.

\title{
Broad-spectrum caspase inhibitors: from myth to reality?
}

\author{
Cell Death and Differentiation (2007) 14, 387-391. doi:10.1038/sj.cdd.4402044; published online 29 September 2006
}

Dear Editor,

The Nomenclature Committee on Cell Death has recently warned the scientific community when using terms such as 'caspase-dependent versus caspase-independent cell death' to classify apoptotic versus nonapoptotic cell death. ${ }^{1}$ We strongly agree with this recommendation and we want to add a word of caution concerning the use of broad-spectrum caspase inhibitors in elucidating apoptotic pathways.

The pioneer work in the 1960s by Dr. Robert Smith has established proteolysis by cysteinyl proteinases as a major physiological and pathological process. ${ }^{2}$ To date, 14 mammalian caspases have been identified from the discovery in 1993 that the Caenorhabditis elegans ced-3 gene encoded a homolog of the human interleukin- $1 \beta$-processing enzyme. ${ }^{3} \mathrm{~A}$ striking feature of caspases is their specific ability to recognize particular short peptide sequences and to cleave after an aspartate residue, which is unique among mammalian proteases (except for the serine protease granzyme B). The introduction of an aldehyde group at the C-terminus of such sequences resulted in the generation of reversible inhibitors, whereas chloromethylketone, diazomethylketone, acyloxymethylketone, fluoromethylketone or phenoxymethylketone at this position created inhibitors that irreversibly inactivated caspases through a covalent thioether adduct with the cysteine of the active site. ${ }^{2,4-9}$ First generations of peptidic 'pan-caspase' inhibitors and caspase activity probes were developed in the 1990s by Enzyme Systems Products. These 